Check for updates

Cite this: RSC Adv., 2018, 8, 3768

Received 17th November 2017 Accepted 12th January 2018

DOI: $10.1039 / c 7 r a 12539 a$

rsc.li/rsc-advances

\section{A rapid and simple UPLC-MS/MS method using collagen marker peptides for identification of porcine gelatin}

\author{
Shangwei Guo, ${ }^{\text {ab }}$ Xiaobing $\mathrm{Xu}^{\mathrm{a}}$ Xiangshan Zhou ${ }^{\mathrm{b}}$ and Yaqin Huang $\mathbb{D}$ *a
}

Gelatin, which is mainly derived from bovine and porcine sources, has been used in many foods and pharmaceutical products. To ensure the compliance of food products with halal regulations, reliable analytical methods are very much required. In this study, one unique marker peptide for porcine gelatins was selected to develop an UPLC-MS/MS multiple reaction monitoring method. The capability of this method to identify porcine materials was demonstrated by analyzing in-house-made gelatins containing different amounts of porcine gelatins and commercial gelatin products. The adulteration of porcine gelatin could be sensitively detected at a low level of $0.04 \%$. When combined with HPLC and mass spectrometry, this method is an accurate and sensitive quantitative method to identify porcine gelatins. Thus, the strategy can be used to verify halal authenticity of gelatin.

\section{Introduction}

Gelatin is a mixture of polypeptides obtained by the selective hydrolysis of collagen, which is the main protein in the skin, hides, white connective tissues, and bones of animals. Gelatin is widely used in food, cosmetic and pharmaceutical industries because of its distinctive advantages, such as biodegradability, non-toxicity, biocompatibility and low cost. ${ }^{1-3}$ And gelatin can be used as an additive in innumerable food products, such as gummy candies, canned ham, various luncheon meals, corned beef, chicken rolls, jellied beef, margarine, sour cream and cottage cheese., ${ }^{4,5}$

Porcine gelatin is the most widely used ingredient in production of processed foods. But in Islamic countries, porcine gelatin is forbidden. ${ }^{6,7}$ However, the food industry and mass market globalization might allow for cheaper or prohibited ingredients to be used and consequently mislabeling, fraudulent substitution or deceptive labeling can occur. ${ }^{8}$ The best way to ensure the "Muslim" of the final food products is to control the raw materials of food processing. To ensure the compliance of food products with halal regulations and the quality and safety of food, a sensitive and reliable analytical approach is urgently needed to identify and distinguish the animal source of gelatin.

The large similarity in structure and properties of gelatin from different origins makes it difficult to distinguish them by conventional spectroscopic methods. Immunochemical methods have been used to identify the species origin of

\footnotetext{
${ }^{a}$ Beijing Laboratory of Biomedical Materials, Beijing University of Chemical Technology, Beijing 100029, China.E-mail: huangyq@mail.buct.edu.cn

${ }^{b}$ Dong'e E-jiao Co., Ltd, National Engineering Research Center for Gelatine TCM, Liaocheng 252201, China
}

gelatins. ${ }^{8}$ However, the immunochemical method may be affected by the degree of proline hydroxylation, which is important in determining the antigenicity of collagen. ${ }^{\mathbf{9 1 0}}$ With high sensitivity and high taxonomic specificity, polymerase chain reaction (PCR), which is associated with DNA, has been commonly used to track the source of an animal product or detect the possible contamination of other animal tissues. ${ }^{\mathbf{1 1 , 1 2}}$ Yet DNA integrity is destroyed during gelatin processing..$^{\mathbf{1 3 , 1 4}}$

In contrast to DNA, the amino acid sequence of protein is highly consistent in the processing of gelatin. The characteristic amino acid sequence of collagen is a repeating $\mathrm{G}-\mathrm{X}-\mathrm{Y}$ sequence, where $\mathrm{G}$ is glycine, and $\mathrm{X}$ and $\mathrm{Y}$ mostly are proline and hydroxyproline, respectively. ${ }^{15}$ This characteristic amino acid sequence has been used for identification of the animal source for gelatin by mass spectrometric detection of marker peptides after trypsin digestion. ${ }^{\mathbf{1 6 , 1 7}}$ For example, collagen marker peptides, combined with sequence search, can distinguish bovine and porcine gelatin. ${ }^{\mathbf{1 8 , 1 9}}$ In addition, Grundy et al. ${ }^{\mathbf{2 0}}$ reported a method for determining the species of origin of gelatin in foods and pharmaceutical products using LC-MS/MS technique. Sarah et al. ${ }^{21}$ found four peptides (EVTEFAK, LVVITAGAR, FVIER and TVLGNFAAFVQK) were consistently detected in cooked pork meat using MRM techniques. Yilmaz et al. ${ }^{22}$ presented a novel nanoUPLC-ESI-qTOF-MSE workflow technique that has the potential to identify the origin of gelatin in some dairy products such as yoghurt, cheese and ice cream. However, an accurate and sensitive method is necessary to not only identify but also quantify porcine gelatins to solve the problems like mislabeling the source and content of porcine gelatins. Sha et $a .^{23}$ established a method by combining trypsin-catalyzed 180 labeling and high-resolution mass spectrometry to quantify the bovine or porcine gelatins. 
To make the analytical method simple and precise, in this study, we use marker peptides detected by UPLC-MS/MS in MRM mode to develop a new analytical method that allowed us to identify and quantify porcine gelatin content. Moreover, inhouse-made and commercial gelatins were analyzed for the selected marker porcine peptide. Our results clearly demonstrated that this method is a very useful and effective technique for halal authentication of commercial pure gelatin and gelatincontaining processed food products.

\section{Experimental method}

\section{Chemicals and reagents}

Trypsin (sequencing grade) was obtained from Promega (Fitchburg, WI, USA). Formic acid, acetonitrile (Ultraperformance liquid chromatography [UPLC]-grade), were purchased from Thermo Fisher Scientific (Waltham, MA, USA). Ultra high purity water was prepared using a Milli-Q water purification system (Millipore Corporation, Bedford, MA). Syringe filters $(0.22 \mu \mathrm{m})$ were purchased from Millipore (Billerica, MA, USA). All other chemicals and reagents were of the highest grade available. Porcine marker peptide standards were synthesized by Jill Biochemical Co., Ltd. (Shanghai, People's Republic of China). Gelatin samples were prepared in the laboratory.

\section{Sample preparation}

First, $50 \mathrm{mg}$ of the gelatin standard was dissolved in $25 \mathrm{~mL}$ of a $1 \% \mathrm{NH}_{4} \mathrm{HCO}_{3}$ solution (pH 8.0). The solution was filtered through a $0.22 \mu \mathrm{m}$ syringe filter. Then, $200 \mu \mathrm{L}$ of the gelatin solution was drawn, and $10 \mu \mathrm{L}$ of trypsin solution $\left(1 \mathrm{mg} \mathrm{mL}^{-1}\right.$ in $1 \% \mathrm{NH}_{4} \mathrm{HCO}_{3}, \mathrm{pH} 8.0$ ) was added. The mixture was incubated at $37^{\circ} \mathrm{C}$ for $12 \mathrm{~h}$.

\section{Chromatographic separation}

The UPLC analysis was performed using a Agilent 1290 Infinity Ultra Performance LC system. Chromatographic separation was carried out on an ACQUITY UPLC BEH C18 column $(100 \mathrm{~mm} \times$ $2.1 \mathrm{~mm}, 1.7 \mu \mathrm{m})$ at a column temperature of $45^{\circ} \mathrm{C}$. The mobile phase consisted of solvent $\mathrm{A}(0.1 \%$ formic acid in acetonitrile, $\mathrm{v} /$ $\mathrm{v})$ and solvent $\mathrm{B}(0.1 \%$ formic acid in water, $\mathrm{v} / \mathrm{v})$. The optimized UPLC elution conditions were as follows: $0.0-25.0 \mathrm{~min}, 5-17 \%$ B; 25.0-26.0 min, 17-99\% B; 26-35 min, 99\% B $\rightarrow$ 99\% B; 35$36 \mathrm{~min}, 99 \% \mathrm{~B} \rightarrow 2 \% \mathrm{~B} ; 36-45 \mathrm{~min}, 2 \% \mathrm{~B}$. The flow rate was set at $300 \mu \mathrm{L} \min ^{-1}$. The injection volume was $5 \mu \mathrm{L}$.

\section{Mass spectrometry}

Mass spectrometry was performed on a Thermo Scientific ${ }^{\mathrm{TM}}$ TSQ Quantum Access Max Triple Quadrupole Mass Spectrometer (with Electrospray Ionization Source HESI). The MS scan were performed over $\mathrm{m} / \mathrm{z}$ ranges of 200-1500. For positive electrospray modes, the spray voltage was set at $3.2 \mathrm{kV}$. The pressure of the sheath gas $\left(\mathrm{N}_{2}\right)$, auxiliary gas (Aux gas, $\mathrm{N}_{2}$ ) and collision (Ar) were set to 40 au (arbitrary units), 5 au and 1.5 mTorr. The temperature of transmission capillary was set to $300{ }^{\circ} \mathrm{C}$.

\section{MRM analysis of marker peptides}

Marker peptides were monitored by LC-MS/MS in MRM mode. The sample solution was loaded onto an BEH C18 column $(100 \mathrm{~mm} \times 2.1 \mathrm{~mm}, 1.7 \mu \mathrm{m})$ at a flow rate of $300 \mu \mathrm{L} \mathrm{min}{ }^{-1}$. Mobile phase A was $0.1 \%$ formic acid in acetonitrile, and mobile phase B was $0.1 \%$ formic acid in water. Quantitations were performed in multiple reaction monitoring (MRM) mode at $3.2 \mathrm{kV}$ multiplier voltage, and the following MRM transitions of $[\mathrm{M}+2 \mathrm{H}]^{2+}$ precursor ions $\rightarrow$ product ions were selected for the marker peptides and the relative collision energies optimized by the Quantum Tune Master® software:

$$
\begin{aligned}
& m / z 473.0 \rightarrow 586.1+717.2(\text { collision energy, } 26 \mathrm{eV} \text { ) } \\
& m / z 774.3 \rightarrow 977.8+809.3 \text { (collision energy, } 30 \mathrm{eV} \text { ) } \\
& m / z 926.0 \rightarrow 833.1+1012.5 \text { (collision energy, } 35 \mathrm{eV} \text { ) }
\end{aligned}
$$

\section{Database searching}

Collagen sequences, including both COL1A1 and COL1A2 from horse, cattle, sheep, tilapia and pig, were downloaded from the National Center for Biotechnology Information (NCBI) website (https://www.ncbi.nlm.nih.gov/protein/, GI: 953866084, 149705490, 75775290, 151555719, 965959815, 426227338, 359804080, 359804082, 1159729721 and 1159729723), which constituted the database we built. The protein sequences from different animal species were compared using the BioEdit Sequence Alignment Editor software.

\section{Results and discussion}

\section{Study design}

A highly sensitive UPLC-MS/MS multiple reaction monitoring strategy was developed, as presented in Fig. 1. First, a variety of gelatins on trypsin digestion were detected by mass spectrometry. Next, marker peptides representing porcine gelatin was selected. Then the suitability for identifying porcine gelatins and detecting possible adulteration was evaluated by MRM.

\section{UPLC-MS analysis of the digested gelatins}

Fig. 2 shows the total ion chromatograms (TIC) of the five gelatins digested at $37^{\circ} \mathrm{C}$ for $12 \mathrm{~h}$ with scan range of $\mathrm{m} / \mathrm{z} 200-$ 1500. The elution profiles indicate that the retention time scale of the peptides in the digested porcine gelatins are similar to that of the peptides in the other digested gelatins. This might result from the high protein homology among the herbivorous mammals. Peptides in the digested samples generally show similar hydrophobicity/hydrophilicity due to the high content of glycine and proline..$^{10,19}$ It is difficult to distinguish each gelatin by visual observation of their chromatograms, therefore, we get EIC (Extracted Ion Chromatogram) from scan range of $\mathrm{m} /$ $z$ 200-1500 to further analysis.

\section{Selection of the marker peptide}

The ion, $\mathrm{m} / \mathrm{z} 473.0,774.0$ and 926.0, were found in the digested the porcine gelatins (Fig. 3). Its MS/MS spectrum 


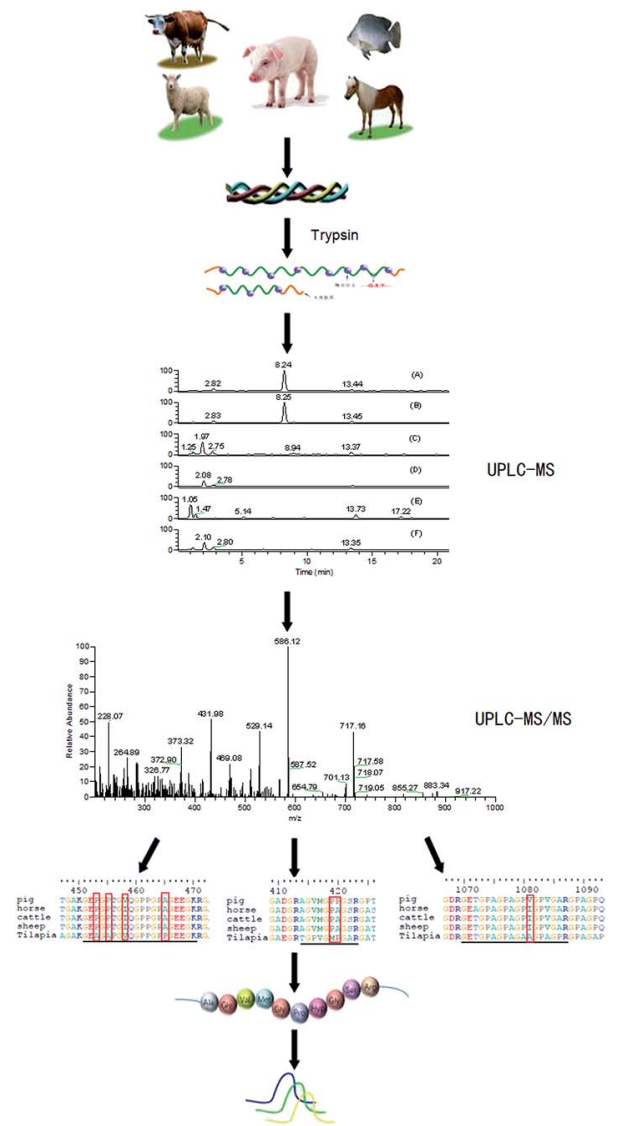

Fig. 1 Strategy used to discover and validate marker peptides for identifying porcine gelatin.

corresponded to the sequence, AGVMGPOGSR (O indicates Hyp, Fig. 4a), GETGPAGPAGPVGPVGAR (Fig. 4b), GEOGPTGVQGPOGPAG EEGK (O indicates Hyp, Fig. 4c), respectively. The sequence located in the a2(I) chain of porcine collagen. These peptides were not found neither in the digested other four gelatins, nor by searching against collagens from all five animal species. The ions with $\mathrm{m} / \mathrm{z}$ values corresponding to marker peptides were extracted from each LC-MS/MS run, and their $\mathrm{m} / \mathrm{z}$ values, charge states, and retention times are summarized in Table 1 . The marker peptides for skin were selected based on three criteria. ${ }^{17}$ First, the peptide can be detected consistently during multiple repetitive runs. Second, the ideal length of the peptide should range from 7 to 15 amino acid residues, making it feasible for the MRM method. Third, the most intensive signal of peptide should be selected in order to simplify the method. Thus, the most abundant peptide, AGVMGPOGSR, in the digested porcine gelatin was used to develop the quantitation method. Our peptide is complete different from the marker peptide sequences (GIpGEFGLPGPAGPR) Kleinnijenhuis et $a .^{24}$ found. By blast search, AGVMGPOGSR is only found in pigs and some other animals that not can be used as raw materials for gelatin so that the porcine marker peptide AGVMGPOGSR has a higher exclusivity for the detection of porcine gelatin.
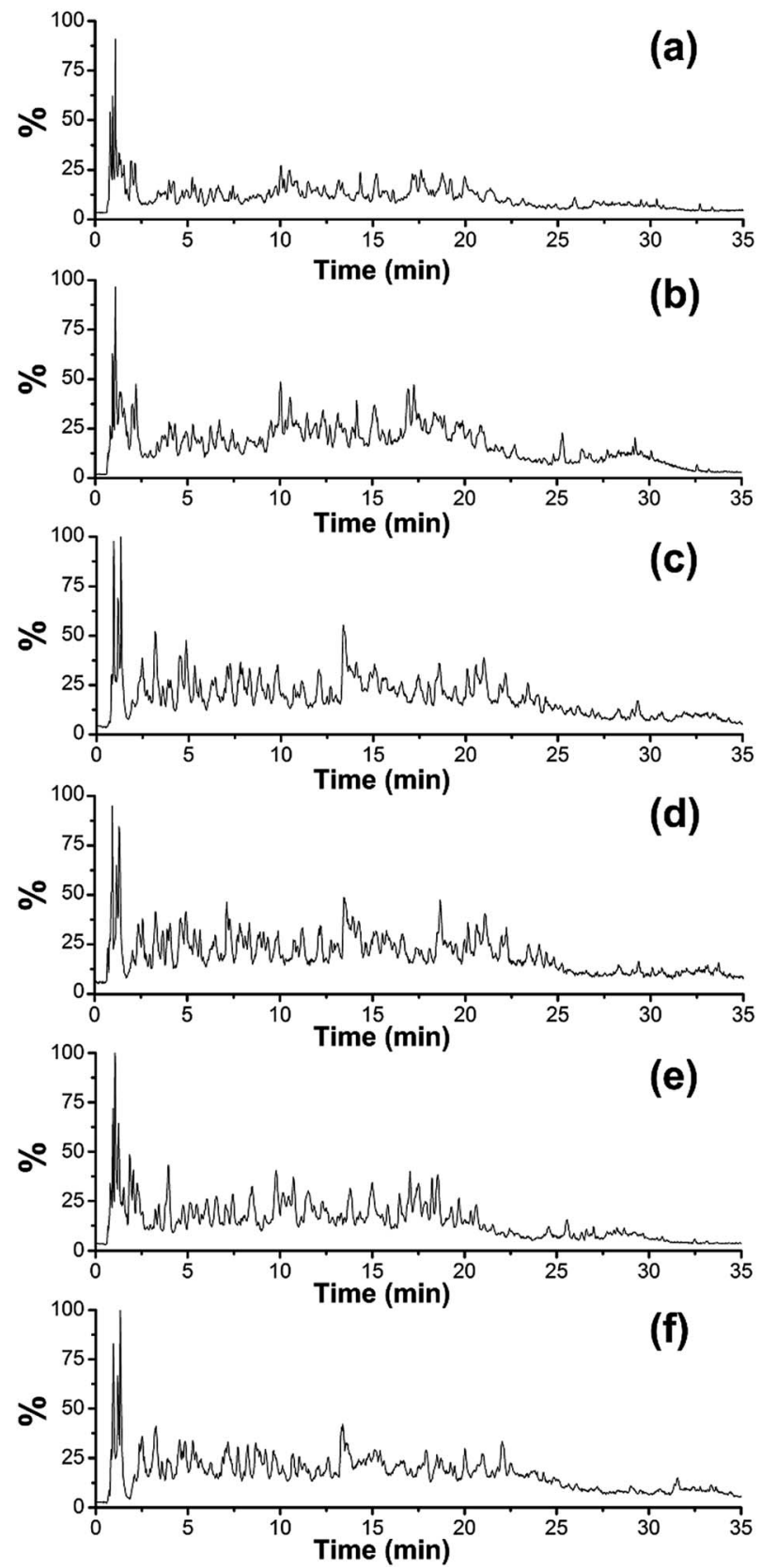

Fig. 2 Positive ion base peak intensity chromatograms obtained from the analysis of tryptic digest of (a) pig-hide gelatin, (b) pig-bone gelatin, (c) cattle-hide gelatin, (d) sheep-bone gelatin, (e) tilapia-hide gelatin, and (f) horse-hide gelatin.

\section{Development of the MRM quantitation method for marker peptides}

MRM experiments were performed on a triple-quadrupole mass spectrometer. Typically, multiple transitions are required to quantify a peptide from complex samples, whereas a single transition may be sufficient for monitoring peptides from particular proteins with high abundance. Collagen is the dominant protein in animal skins and bones. Three or more transitions were tested for each marker peptide and the most abundant ones were selected in order to simplify the method. 


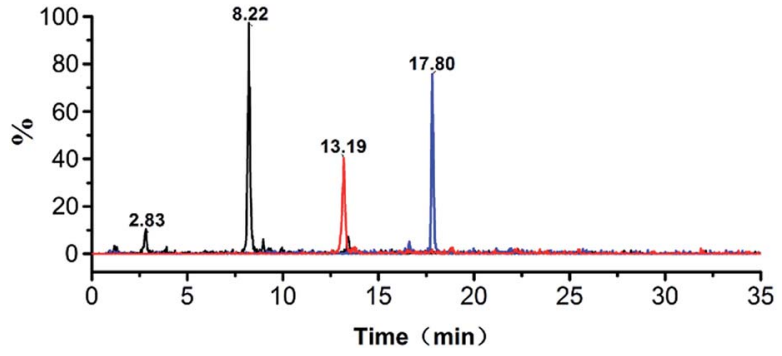

Fig. 3 Extracted ion chromatogram obtained from the analysis of tryptic digest of porcine gelatin.
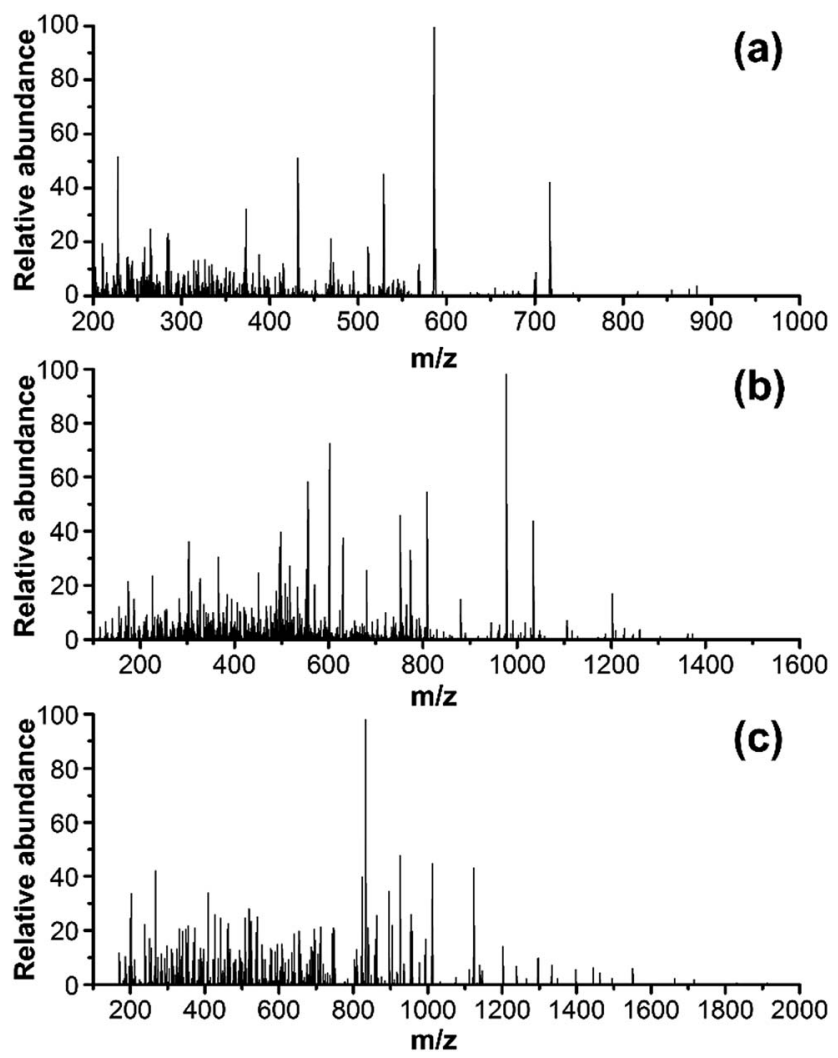

Fig. 4 MS/MS spectra of one marker peptide for porcine gelatins: (a) peptide $\mathrm{PM} 1, \mathrm{~m} / \mathrm{z}=473.0$, doubly charged; (b) peptide $\mathrm{PM} 2, \mathrm{~m} / \mathrm{z}=$ 774.3, doubly charged; and (c) peptide PM3, $m / z=926.0$.

Transitions $473.0 \rightarrow$ 586.1, 774.3 $\rightarrow$ 977.8, 926.0 $\rightarrow$ 833.1 were tested for monitoring PM1, PM2 and PM3, and 473.0 $\rightarrow 586.1$ exhibited the most intensive signal. The MRM chromatograms of gelatins prepared from pig shown in Fig. 3. The marker peptides PM1 was eluted at $8.22 \mathrm{~min}$. The identity of porcine gelatins was revealed unambiguously by the presence of

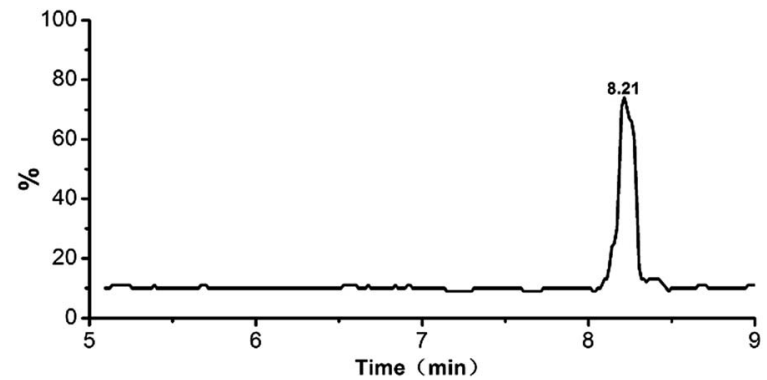

Fig. 5 MRM chromatograms of porcine gelatins.

corresponding marker peptides. The limit of quantitation was $0.10 \mathrm{pg}$ (Fig. 5).

Next, this marker peptide was synthesized to develop a quantitation method. Solutions of the peptide with various concentrations, ranging from $0.1 \mu \mathrm{g} \mathrm{mL}{ }^{-1}$ to $8 \mu \mathrm{g} \mathrm{mL}{ }^{-1}$, were prepared. The concentrations were analyzed in triplicate, and 2 $\mu \mathrm{L}$ of sample was injected for LC-MS/MS MRM run. As shown in Fig. 6, standard curve was plotted using the MRM peak area against the amount of sample injected. Good linearity was obtained, and $R^{2}$ value was greater than 0.999 .

\section{Marker peptide detection in gelatins}

To demonstrate the ability of this MRM method to identify porcine gelatins in gelatins, the horse-hide gelatin was mixed with $1 \%, 5 \%, 40 \%$ of porcine gelatins, respectively. Sample preparation was simplified to adapt this method for use in routine applications. Triplicates were analyzed for each sample, and the representative MRM chromatograms are shown in Fig. 7a-c. When porcine gelatin was added, the marker peptide for porcine gelatin was observed. The addition of porcine gelatins was easily detected at an amount over $1 \%$ of the total weight in repetitive runs. These results suggest that this method is highly sensitive and is able to detect a trace amount of porcine gelatins added.

\section{Optimization of enzymolysis time}

In order to separate all of characteristic polypeptide by enzymolysis, detected the samples at $0,4,6,8,9,12,14 \mathrm{~h}$ after added trypsin for finding out how much time the enzymatic process took. The results showed the peak area under ion pair $473.0 \rightarrow$ 586.1 was almost no change between 8-14 hours. The data indicates that the characteristic polypeptides are completely separated by trypsin solution after 8 hours. The suitable testing conditions including enzyme, enzymatic hydrolysis ability and enzymatic time are key points for pretreatment. Consequently,

Table 1 List of selected marker peptides

\begin{tabular}{|c|c|c|c|c|c|}
\hline Marker peptide & Sequence & Protein & Charge state & $m / z$ & Precursor mass \\
\hline PM1 & ${ }^{414} \mathrm{AGVMGPOGSR}^{423}$ & COL1A2 & 2 & 473.0 & 944.08 \\
\hline PM2 & ${ }^{1069}$ GETGPAGPAGPVGPVGAR $^{1086}$ & COL1A1 & 2 & 774.3 & 1546.70 \\
\hline PM3 & ${ }^{451}$ GEOGPTGVQGPOGPAGEEGK ${ }^{470}$ & COL1A1 & 2 & 926.0 & 1849.93 \\
\hline
\end{tabular}




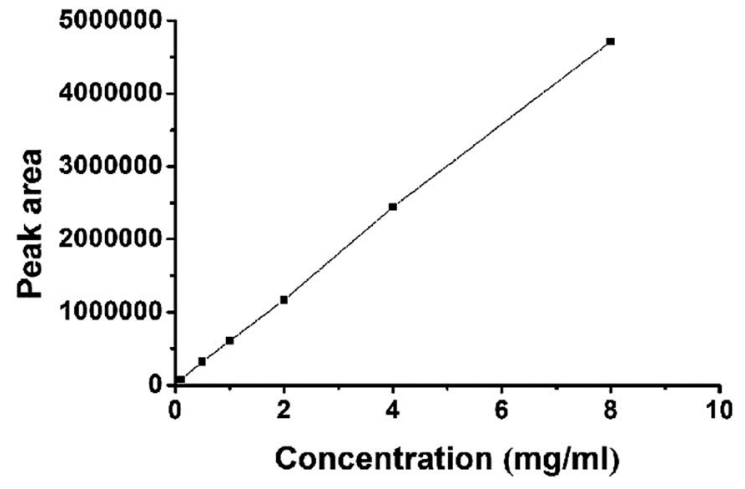

Fig. 6 Linearity of synthetic marker peptide.
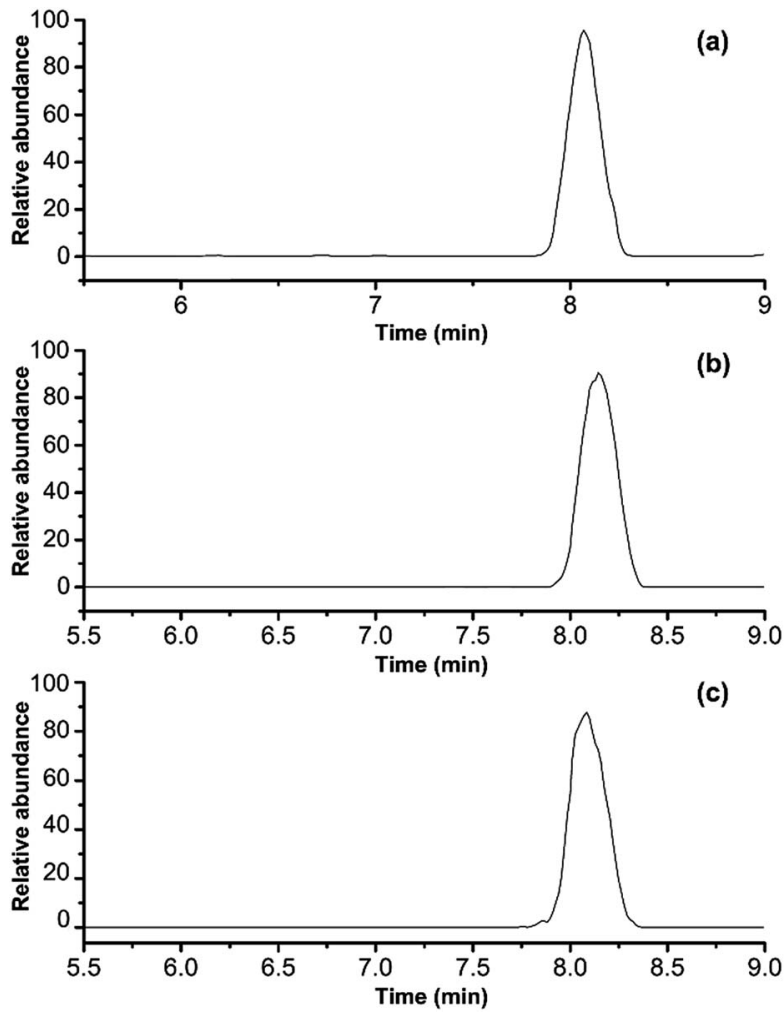

Fig. 7 MRM chromatograms of porcine gelatins in different concentration: (a) $1 \mathrm{wt} \%$, (b) $5 \mathrm{wt} \%$, and (c) $40 \mathrm{wt} \%$.

the enzymolysis time of gelatin with trypsin solution can be set to 8 hours or more.

\section{Conclusions}

A UPLC-MS/MS multiple reaction monitoring method with highly sensitive has been successfully used to distinguish porcine gelatins in different products. The UPLC-MS/MS detection coupled with MRM can profile details of the samples so that important markers of porcine gelatin can be measured even at low concentration levels. The marker peptides of gelatins can be used to identify porcine gelatins in a mixture.
As a result, the strategy can be used to verify halal authenticity of gelatin.

\section{Conflicts of interest}

There are no conflicts to declare.

\section{Acknowledgements}

Financial supported from the Fundamental Research Funds for the Central Universities (No. PYBZ710) is gratefully appreciated.

\section{References}

1 G. Li, S. Fukunaga, K. Takenouchi and F. Nakamura, Int. J. Cosmet. Sci., 2005, 27, 101-106.

2 M. Hamidi, A. Azadi and P. Rafiei, Adv. Drug Delivery Rev., 2008, 60, 1638-1649.

3 Y. Liu and M. B. Chan-Park, Biomaterials, 2009, 30, 196-207.

4 T. Tasara, S. Schumacher and R. Stephan, J. Food Prot., 2005, 68, 2420-2426.

5 M. Czerner, L. A. Fasce, J. F. Martucci, R. Ruseckaite and P. M. Frontini, Food Hydrocolloids, 2016, 60, 299-307.

6 H. I. A. Amqizal, H. A. Al-Kahtani, E. A. Ismail, K. Hayat and I. Jaswir, Food Control, 2017, 78, 297-303.

7 A. Karim and R. Bhat, Trends Food Sci. Technol., 2008, 19, 644-656.

8 R. S. Sawhney, Cell Biol. Int., 2005, 29, 133-137.

9 A. Venien and D. Levieux, J. Pharm. Biomed. Anal., 2005, 39, 418-424.

10 X.-L. Cheng, F. Wei, X.-Y. Xiao, Y.-Y. Zhao, Y. Shi, W. Liu, P. Zhang, S.-C. Ma, S.-S. Tian and R.-C. Lin, J. Pharm. Biomed. Anal., 2012, 62, 191-195.

11 O. Fumière, M. Dubois, V. Baeten, C. von Holst and G. Berben, Anal. Bioanal. Chem., 2006, 385, 1045.

12 J. S. Amaral, G. Santos, M. B. P. Oliveira and I. Mafra, Food Control, 2017, 72, 53-61.

13 M. Merheb, S. Vaiedelich, T. Maniguet and C. Hänni, Mitochondrial DNA, Part A, 2016, 27, 355-359.

14 K. Tasanen, R. Palatsi and A. Oikarinen, Br. J. Dermatol., 1998, 139, 23-26.

15 K. Pawelec, S. M. Best and R. Cameron, J. Mater. Chem. B, 2016, 4, 6484-6496.

16 G. Zhang, A. Sun, W. Li, T. Liu and Z. Su, J. Chromatogr. A, 2006, 1114, 274-277.

17 X. Li, F. Shi, L. Gong, B. Hang, D. Li and L. Chi, Int. J. Nanomed., 2017, 12, 4443.

18 G. Zhang, T. Liu, Q. Wang, L. Chen, J. Lei, J. Luo, G. Ma and Z. Su, Food Hydrocolloids, 2009, 23, 2001-2007.

19 M. Nemati, M. Oveisi, H. Abdollahi and O. Sabzevari, J. Pharm. Biomed. Anal., 2004, 34, 485-492.

20 H. H. Grundy, P. Reece, M. Buckley, C. M. Solazzo, A. A. Dowle, D. Ashford, A. J. Charlton, M. K. Wadsley and M. J. Collins, Food Chem., 2016, 190, 276-284. 
21 S. A. Sarah, W. N. Faradalila, M. S. Salwani, I. Amin, S. A. Karsani and A. Q. Sazili, Food Chem., 2016, 199, 157-164.

22 M. T. Yilmaz, Z. Kesmen, B. Baykal, O. Sagdic, O. Kulen, O. Kacar, H. Yetim and A. T. Baykal, Food Chem., 2013, 141, 2450-2458.
23 X. M. Sha, Z. C. Tu, H. Wang, T. Huang, D. L. Duan, N. He, D. J. Li and H. Xiao, J. Agric. Food Chem., 2014, 62, 1184011853.

24 A. J. Kleinnijenhuis, F. L. van Holthoon and G. Herregods, Food Chem., 2018, 243, 461-467. 